International Journal of Distributed and Parallel Systems (IJDPS) Vol.3, No.1, January 2012

\title{
COMMON CONTROL CHANNEl SATURATION DETECTION AND ENHANCEMENT IN COGNITIVE RADIO NETWORKS
}

\author{
Seyed Morteza Mirhoseni Nezhadal ${ }^{1}$, Reza Berangi ${ }^{2}$ and Mahmood Fathy ${ }^{3}$ \\ ${ }^{1}$ Department of Computer Engineering, Iran University of Science \& Technology, \\ Tehran, Iran \\ 1 mortaaza@gmail.com \\ ${ }^{2}$ rberangidiust.ac.ir \\ 3 mahfathyeiust.ac.ir
}

\begin{abstract}
Mac protocol plays an important role in spectrum opportunities utilization, primary user's interference management and secondary user's coordination in cognitive radio networks. The Common control channel is one of the most challenging issues in CR networks. This channel is regarded as bottleneck for the network and any threat for it can be assumed as a threat for the whole network. CCC saturation is one of the most important problems, when the number of users in the network is high.

In this paper we are going to propose a method to diagnose CCC saturation. After saturation detection $M A C$ decision can select an alternative strategy for user's coordination. We introduced through CCC access scheme (IEEE 802.11) an analytical model to describe our method. According to this method in CCC saturation situation the network throughput not highly decreased and not huge increasing in transmission delay. So using this method can avoids waste of CR network resources considerably.
\end{abstract}

\section{KEYWORDS}

Opportunistic spectrum access, Cognitive Radio Networks (CR networks), Common Control Channel (CCC), Medium Access Control (MAC).

\section{INTRODUCTION}

Today, frequency spectrum allocation is done by governmental agencies. These organizations allocate part of spectrum to real or legal users statically. Generally major part of this allocated bandwidth is seldom used. Although this allocation strategy was useful in the past, dramatic increase in the number of wireless service users has made the previous spectrum allocation strategies useless. The spectrum scarcity in one hand and none optimal usage of available spectrum in the other hand, we have to use methods to increase spectrum efficiency [1].

As you see in the figure 1, there can be a large variation in spectrum use intensity, utilization of spectrum in some frequencies are dense whereas large amount of spectrum remain useless. Solution for solving this problem is to use dynamic spectrum access algorithms [2], [3].

After spectrum sensing in physical layer, a channel will be assigned to applicant secondary users by MAC protocol if possible. Before two secondary user's connection in CR network, they need to meet on a channel that is available for both of them, in order to set up a communication link. 
So using Common control channels would be helpful. Definition and implementation of CCC is one of the most challenging issues is CR networks, it has been called CCC problem [4], [18].

In the next chapter, we will review the history of CCC implementation in different MAC protocols. Chapter 3 describes proposed method. In chapter 4 offers suggested analyze method and compression with diagrams. And finally, there will be conclusion of what we have reviewed in this paper.

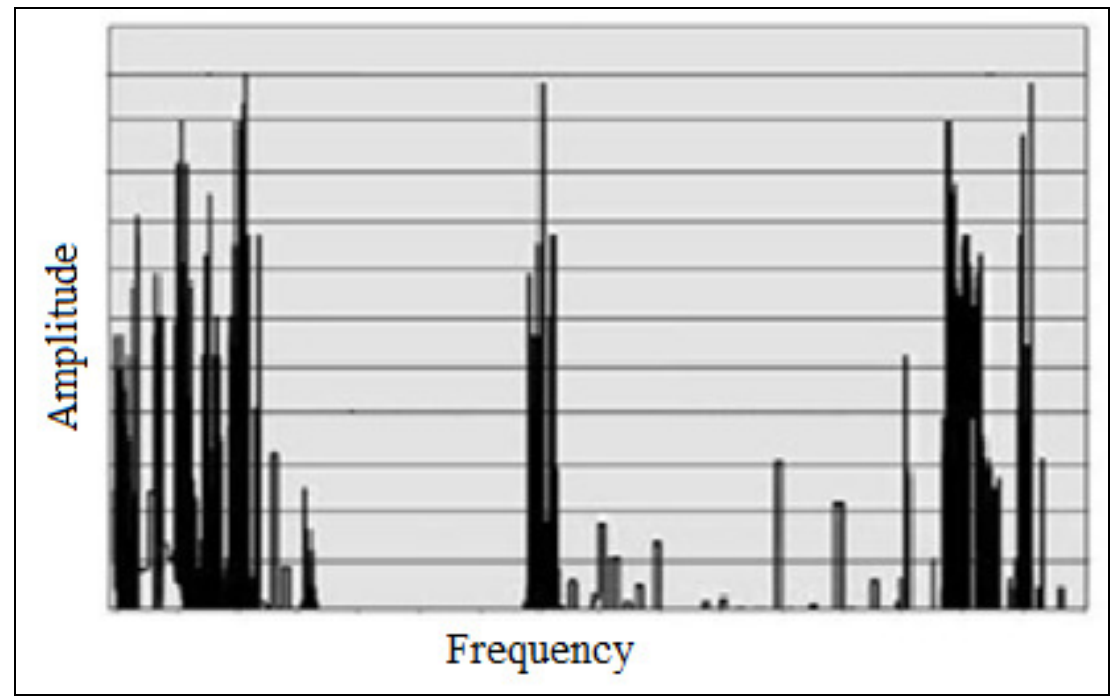

Figure 1. Spectrum efficiency [2]

\section{Previous Works}

Authors in [5] assumed CCC as part of licensed band; it means all control information will be exchanged among secondary users via that dedicated CCC and data communication will take place on other channels. So it is clear that for this assigned channel there is no risk of primary user coming back, but it has its own problems such as saturation and security problems. The other proposed solution is to assume CCC through unlicensed available channels. [7] , [6] this solution also has some challenges, unreliability and impossibility of determining unique CCC for entire network which endanger network

Jia and Zhang proposed a MAC protocol which reviews hardware limitations such as transmission and receive restrictions. It has been analyzed by formulating receiving stop, problem and its optimization that results better output for CR network and improves spectrum throughput. In this protocol a dedicated common channel which is accessible by RTS/CTS messages, has been assumed for exchanging control information, [8].

Authors in [9], [10] and [11] has proposed MAC protocol regardless of common channels. they have utilized internal handshaking mechanisms to coordinate transmission parameters.

B.Hamdaoui and his partners proposed OS-MAC protocol for CR networks in which traffic load will be balanced between frequency bands, spectrum access will be done fairly between secondary users and control overload is very low and cognitive user's reconfiguration is based on network current traffic load. The CCC is assumed as dedicated channel and its access is not through RTS/CTS and decisions will apply as a group to the network. It should be stated that only one transmitter receiver is assumed for this protocol. 


\section{SYSTEM MODEL}

In this section we will review network and radio architecture for communication.

The channels which are not occupied by licensed users can be used by cognitive radio. Before any data exchange, secondary users should meet each other, if negotiation occurs on control channel, it will be done by sending RTS/CTS packets.

The Transmitter sends information including available channels through RTS, receiving RTS, receiver selects suitable channel and put it in CTS and send it to the transmitter.

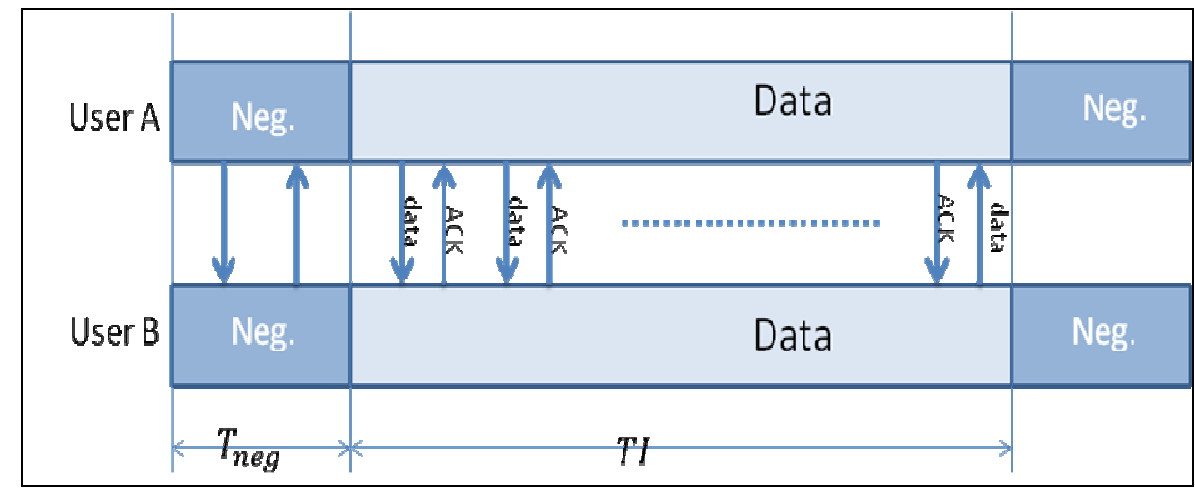

Figure 2. Negotiation and communication timing of secondary users

As shown in figure 2, before any data transmission, two user need to make agreement on transmitting parameters. Main purpose of negotiation is to find common channel for data transmission. After negotiation, users will exchange information in TI period through agreed channel. This Period should be so long that if the primary user appeared, it could tolerate resulting error caused by interference.

\section{Common Channel ACCESS}

There is a permanent common control channel for secondary users with no risk of primary users presence through it (there is no need to know about this channel details).

For every pair of transmitter/ receiver in any period or TI, only 2 RTS/CTS will be sent through CCC. Time intervals are not synchronized with each other and each user defines communication period according to an algorithm. If the user had no packet to send, it would listen to the CCC for a while and then move to another phase as follow. The user responds to receiving packet according to the request.

\section{Control Channel Random Access}

At first, Access to control channel is as IEEE 802.1- DCF.[13] in which each user having packet to send, after sensing phase, decides to act as follow. If the spectrum is available and this status remains the same as DIFS, the user will send RTS over CCC. Otherwise, if the spectrum is busy it will continue sensing till finding it available for DIFS. Here the definition of busy is different. The CCC is not busy when at least it has on licensed free channel; otherwise, the spectrum is called busy. Even if the CCC is free, at least no channel (licensed) is free.

After free spectrum detection, the user generates a backward random number. This will reduce probability of collision with other user's sent packets. DCF uses backward discrete time to 
increase performance. Time is divided to some slots and any probable sending action takes place at the beginning of each time slot.

In each packet sending action, backward timer selects random number from [0, w-1] with equal probability (p). $\mathrm{W}$ indicates competition window. In first attempt $\mathrm{W}$ is equal to $C W_{\min }$, after each unsuccessful transmission it becomes double, until it reaches its maximum value, $C W_{\text {min }}=2^{m} C W_{\min }$.

According to figure 3, the user provided with transmission conditions, uses above mentioned backward rule, and after the backward timer shows zero, RTS packet will be sent to the receiver. If the receiver detects RTS, the CTS packet will be sent to the transmitter after SIFS period. After this phase, two involved SU will change their channel to the agreed channels. After TI finishes, Transmitter/Receiver go to the CCC and there again begins the competition for taking the channel

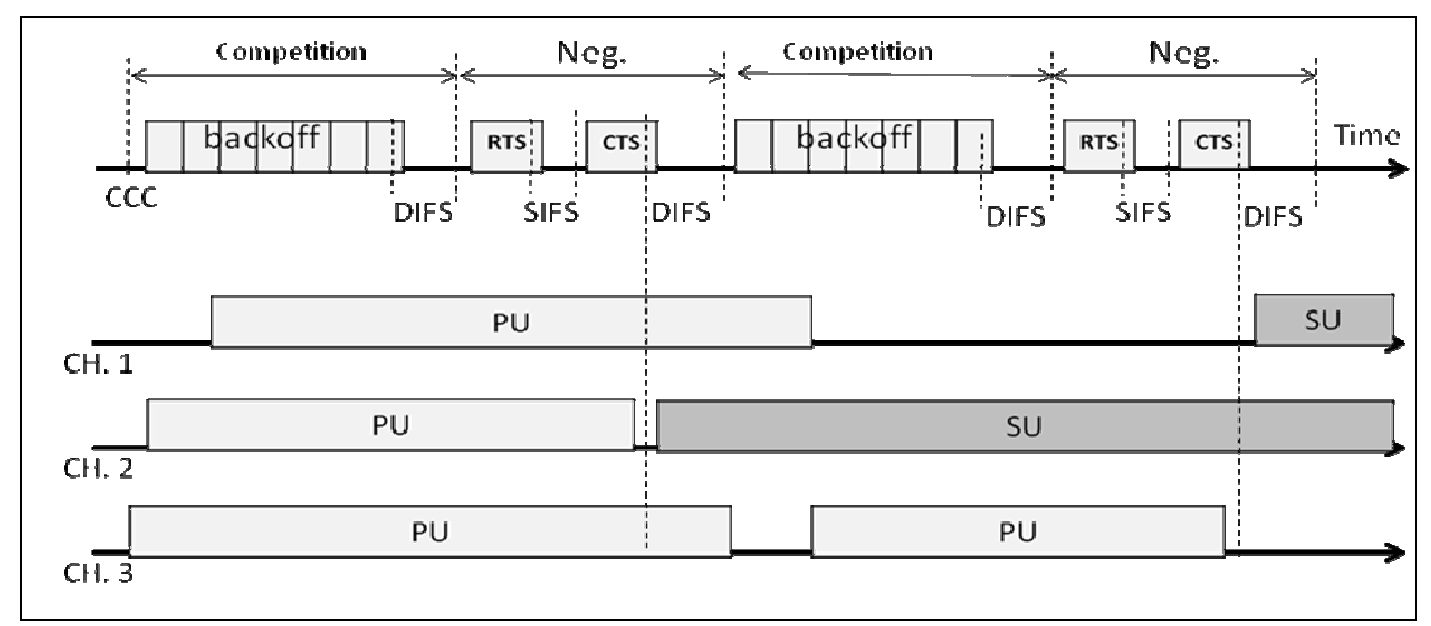

Figure 3. User's random access according to DCF

In the case that, the number of licensed channel and secondary users are high, CCC will suffer repetitious reference. Raising the number of users who want to take control channel causes a rapid growth of competition window size and ultimately increases control channel access time. As you see in the next sections, this event decreases network throughput considerably by increasing negotiation time $T_{\text {neg }}$.

\section{SEQuenCE-BASEd RENDEZVOUS}

In the context of dynamic spectrum access (DSA), rendezvous refers to the ability of two or more radios to meet and establish a link on a common channel.

These will enable radio mediums find each other without having CCC. Time to rendezvous (TTR) is the time which two radio medium visit each other on common frequency. Radios will follow sequences for frequency hopping [14]. 


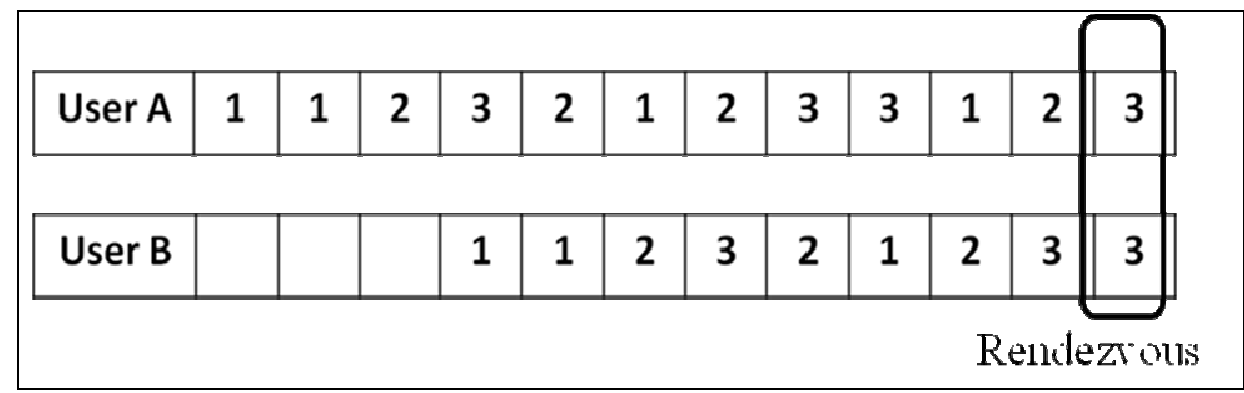

Figure 4. Users A and B come to the rendezvous according to the sequence

Proposes sequence design with good non- orthogonal properties. Each radio assumes a channel sequence which minimizes expected TTR even while radios are not synchronous. E.g. in figure 4, imagine that radio A starts working at $\mathrm{t} 1$ to find radio $\mathrm{B}$ and radio $\mathrm{B}$ dose the same at $\mathrm{t} 2$.

In this method each radio follows a predetermined sequence in which they will visit channels which are potent to be available. Average steps to reach to rendezvous is [14]:

$$
E\left[T T R \_ \text {Stage }\right]=\frac{M^{3}-2 M^{2}+9 M-4}{2(3 M-1)}
$$

M shows the number of channels and indicates that the algorithm order is $M^{2}$.

\section{Detection of Control Channel saturation}

Imagine that $T_{C C C}$ Is the average negotiation time for secondary users on CCC and there is $\mathrm{M}$ channel each relates to one specific PU. Maximum number of competitor Users that can take channel in TI is $N_{s u_{-} \max }$. Obviously, $N_{s u_{-} \max }$ relates to TI inversely so we can say $N_{s u_{-} \max }=\left\lceil T I / T_{C C C}\right\rceil$.

By comparing $\mathrm{M}$ and $T_{C C C}$,it is possible to find out if the control channel has been saturated or not in other word there are still unused channels if $T_{C C C}+1 \leq M$ This means CCC saturation or network bottleneck. Figure 5-3 shows CCC saturation example. 
International Journal of Distributed and Parallel Systems (IJDPS) Vol.3, No.1, January 2012

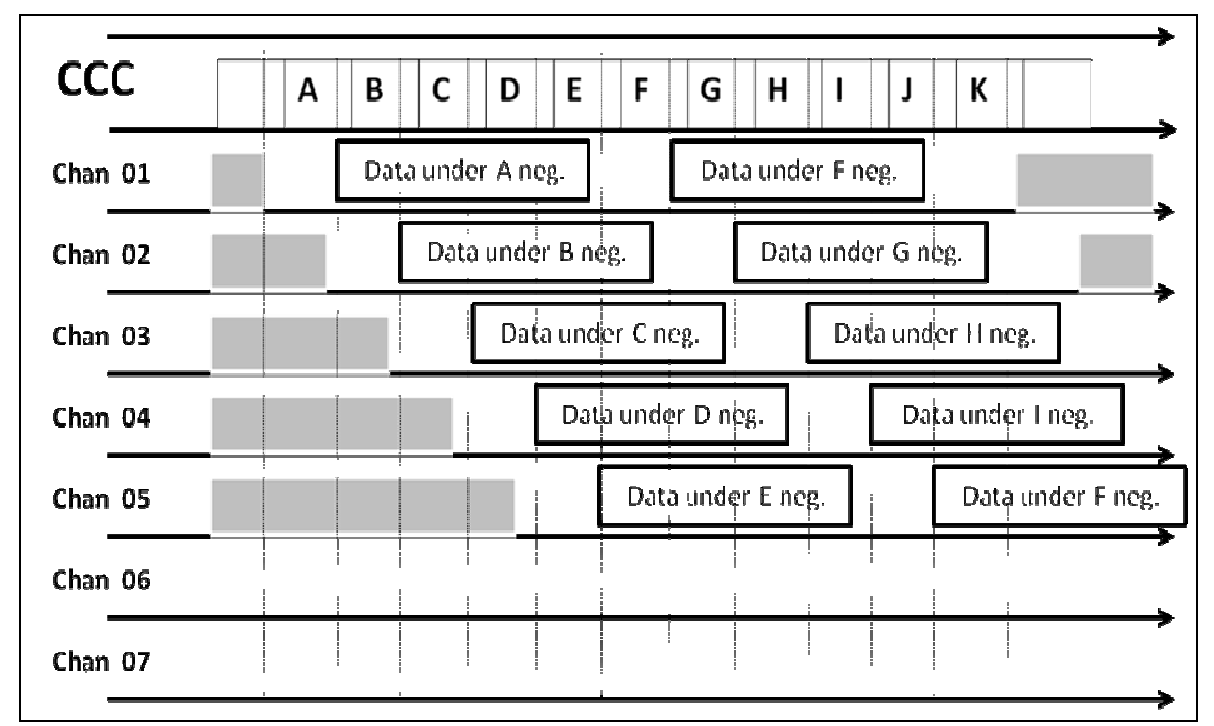

Figure 5. CCC saturation example

In above sample there are 7 channels and $T_{C C C}=4$ so $4+1 \leq 7$ satisfies the saturation condition. Therefore in this example always there are 2 unused channels averagely. you see how CCC bottleneck can disrupt network maximum throughput. So, using sequenced base rendezvous method can be effective here, because meanwhile, the delay caused by control channel is more than rendezvous algorithm. After sensing such situation, the users starts generating sequence based frequencies ([14], [15] provide optimum sequence generation details). As mentioned before, users will find each other after a while and will continue transmission during TI. When SU finds control channel saturated, it will enter into this phase, and its addition to the network throughput rendezvous method delay calculation is really time consuming.. User with data to send will find receiver on common channel after $T_{R}$, and start transmission over agreed channel. But, the user with no data sets its receiver according to the sequence after sensing CCC saturation. So, the negotiation time $T_{n e g}$ might be different according to the network status.

\section{Proposed METhOd ANALYSIS}

To analyze SU's competitive manner for taking CCC,[16] (\& [17]) are used in which IEEE802.11 DCF has been analyzed. $\tau$ Is the probability of one user transmission in random time slot. Collision happens for transmitted packet if at least one another user among n-1 users transmits. So, the probability would be $p=1-(1-\tau)^{n-1} \cdot p_{t r}$ Indicates to have at least one transmission in an assumed time slot. That's because $\mathrm{n}$ users are competing to take control channel and each transmission occurs by probability of $\tau$. So, we have $p=1-(1-\tau)^{n-1}$. Competition 
International Journal of Distributed and Parallel Systems (IJDPS) Vol.3, No.1, January 2012

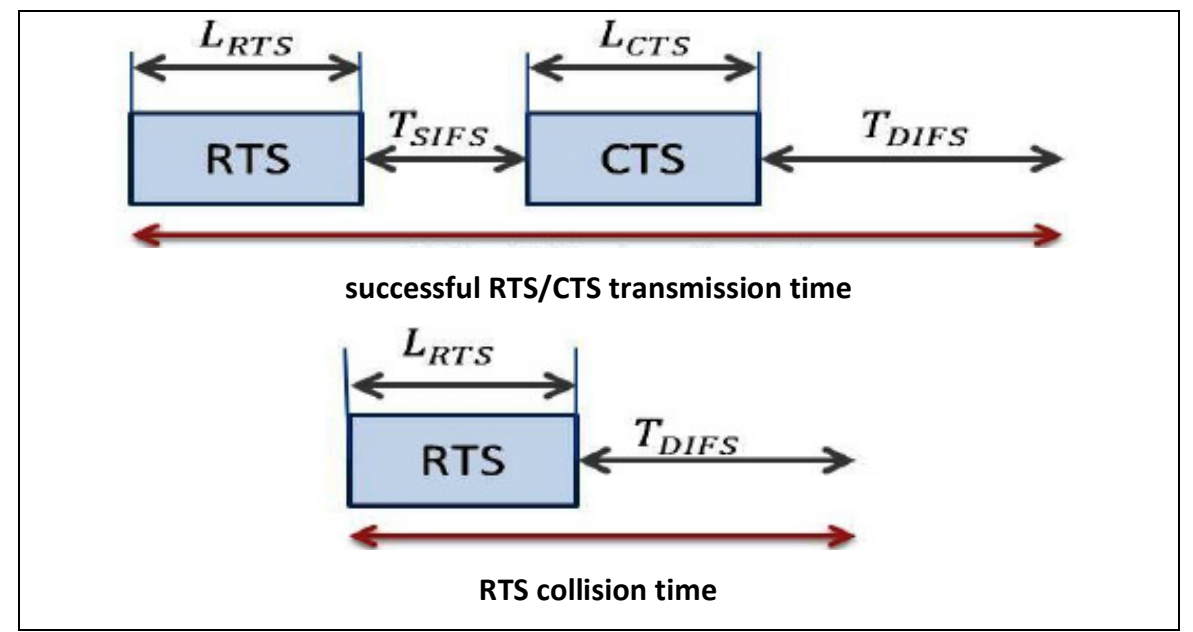

Figure 6. RTS/CTS successful transmission and collision time

$p_{t r}$ Indicates the probability of successful transmission over a channel. The Probability of transmission by one user exactly over a channel is:

$$
P_{s}=\frac{n \tau(1-\tau)^{n}}{P_{t r}}=\frac{n \tau(1-\tau)^{n-1}}{1-(1-\tau)^{n}}
$$

According to figure $6, T_{\text {succ }} \& T_{\text {coll }}$ are as follow:

$$
\begin{aligned}
& T_{\text {succ }}=\left(L_{R T S}+L_{C T S}\right) / R_{C}+T_{S I F S}+T_{D I F S} \\
& T_{\text {coll }}=\left(L_{R T S}\right) / R_{C}+T_{D I F S}
\end{aligned}
$$

In which $R_{c}$ is control channel transmission rate, $L_{R T S} \& L_{C T S}$ indicates RTS and CTS packet length. Also $T_{S I F S} \& T_{D I F S}$ indicates DISF and SIFS time. According to the description above, the negotiation time for RTS and CTS method is as follow:

$$
\begin{aligned}
& T_{C C C}=\frac{\left(1-P_{T R}\right) \sigma+T_{S} P_{t r} T_{\text {succ }}+P_{t r}\left(1-T_{s}\right) T_{\text {coll }}}{P_{s} P_{t r}} \\
& =T_{\text {succ }}+\frac{1-P_{t r}}{P_{s} P_{t r}}+\frac{1-P_{s}}{P_{s}}+T_{\text {coll }}
\end{aligned}
$$

$T_{C C C}+1 \leq M$ indicates network bottle neck or saturation. In addition, $T_{C C C}+1>M$ means that before saturation state that the negotiations delay $T_{n e g}=T_{C C C}$ should be calculated from (4). In this situation, always there is a blank time between two negotiations in CCC. This is the state in which data channels are occupied to the extent that there is users.

Regarding whether the common channel has been saturated or not, throughput calculation will be divided into two phases. In the first phase, the control channel is not yet saturated, and in the second phase, the saturation is detected. In this phase, delay or $T_{n e g}$ contains saturation detection time and finding common channel with rendezvous algorithm. For each phase $T_{n e g}$ is: 
$T_{n e g}=\left\{\begin{array}{l}T_{C C C}\left(N o n \_S a t \_C C C\right) \\ T_{C C C \_n e g}+T_{R}(\text { Saturated_CCC })\end{array}\right.$

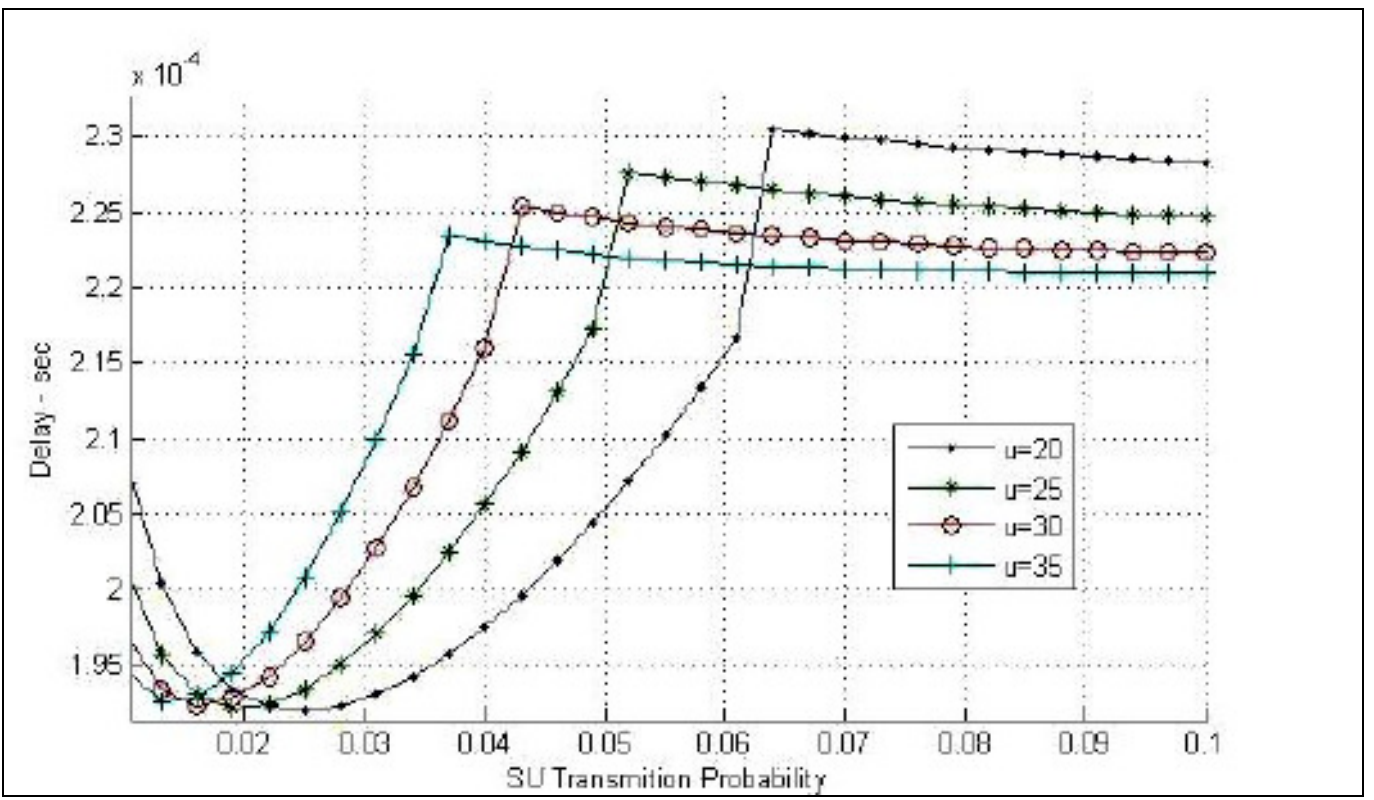

Figure 7. Average negotiation time for $\mathrm{u}$ user in term of $\tau$

According to the previous section, CCC saturation could be defined from the network conditions.

$$
\eta=\frac{T I}{m-1}
$$

As long as channel access is $<T_{C C C_{-} s a t}$, one should not enter into second phase. As soon as exiting from this time interval, the channel will be find through rendezvous method, but $T_{R}$ corresponds to the calculation from (1), and network throughput is:

$$
\eta=\frac{T I \cdot M(1-\tau) R_{d}}{T_{C C C}+T I}
$$

$\mathrm{M}$ is the channel number and $\tau$ is the probability of primary user presence.

As shown in figure 7, the increase in SU's transmission probability, the upward slop caused by negotiation phase is high. CCC saturation point is shown as break point in diagram and rest of points coordinate with each other by rendezvous method, therefore break point emerges as shown in figure 7. Supposedly, it is expected that throughput figure be inverse of delay. As figure 8 shows, more competitor users will results into more throughput decrease second, there will be a break in throughput diagram after CCC saturation detection, then throughput remains almost constant by the increase of $\tau$. 
International Journal of Distributed and Parallel Systems (IJDPS) Vol.3, No.1, January 2012

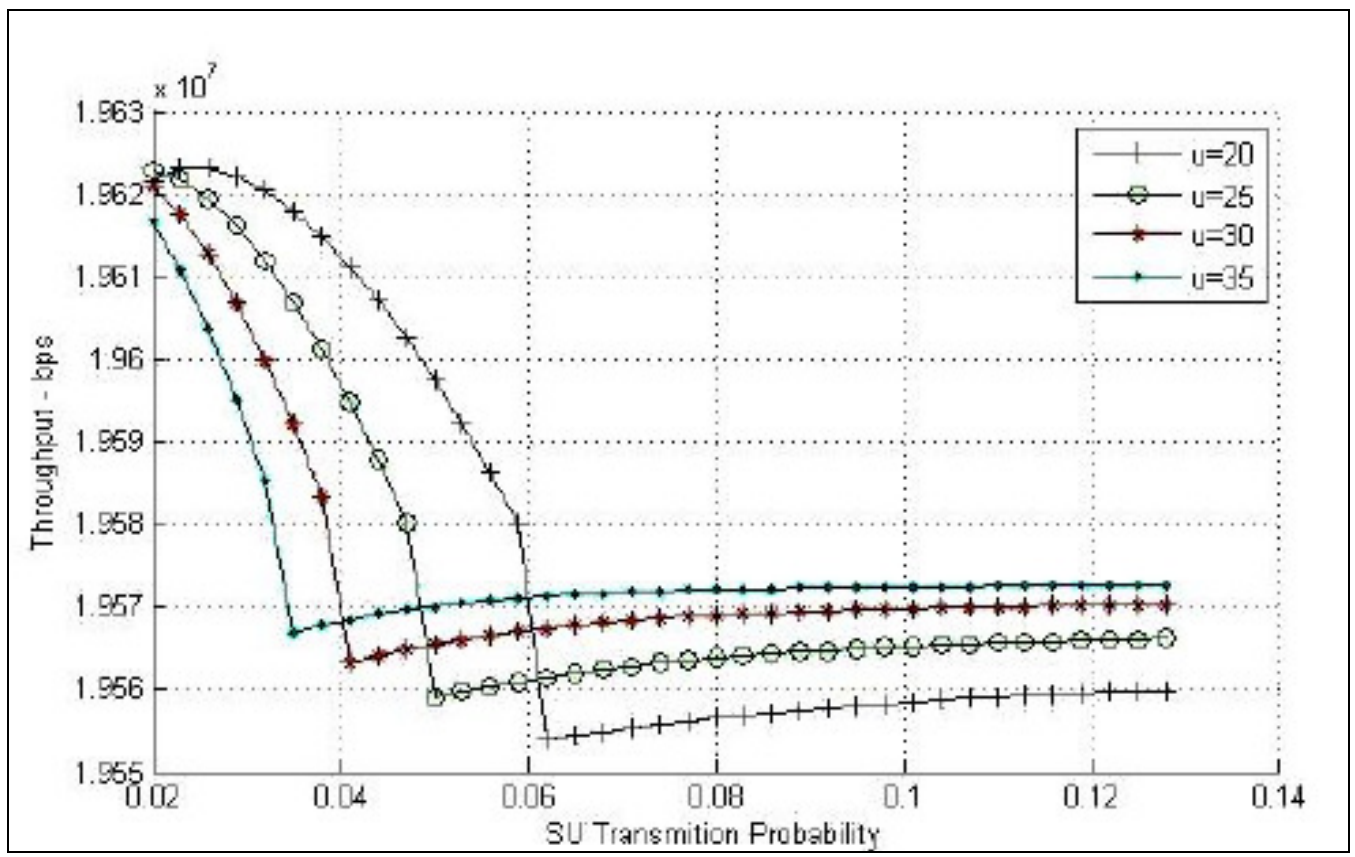

Figure 8. Throughput for u user

\section{Conclusions}

CCC problem is one of the most critical issues in cognitive radios which have been discussed from different aspects in different references.

Control channel saturation in ad-hoc cognitive networks (centralized) is one of the most important ones. In this paper, we tried to propose a new solution to overcome CCC saturation problem. Data transmission of two cognitive users begins after their coordination. This negotiation is thought common control channel. Initially users compete to take CCC by IEEE 802.11 DCF.

If number of competing users was too high, the saturation problem will be appearing. More user in competition process means more time take to catch CCC. After saturation detection another mechanism will run. This method called rendezvous based negotiation. As clarified in mathematical analysis and diagrams, this method has prevented the waste of network resources while CCC saturation.

Other benefit of this method is resistible against DoS attacks, the special kind of attack that lead to increase access time of user to catch CCC. In this position, our method will detect CCC saturation and use alternative method ( User negotiation without CCC).

\section{REFERENCES}

[1] Mitola, J., III; Maguire, G.Q., Jr.; , "Cognitive Radio: Making Software Radios More Personal," Personal Communications, IEEE, vol.6, no.4, pp.13-18, Aug. 1999

[2] Zhao, Q.; Sadler, B.M.; , "A Survey of Dynamic Spectrum Access," Signal Processing Magazine, IEEE, vol.24, no.3, pp.79-89, May. 2007

[3] I.F. Akyildiz, W.-Y. Lee, M.C. Vuran, S. Mohanty, "NeXt generation dynamic spectrum access cognitive radio wireless networks: a survey," Computer Networks Journal (Elsevier), Issue 13, 50, pp. 2127-2159, Sep 2006. 
International Journal of Distributed and Parallel Systems (IJDPS) Vol.3, No.1, January 2012

[4] Xiang, J.; Zhang, Y.; Skeie, T.; , "Medium Access Control Protocols in Cognitive Radio Networks," Wireless Communications and Mobile Computing, vol.10,no.1, pp. 31-49, Nov. 2010

[5] Pawelczak, P.; Venkatesha Prasad, R.; Xia, L.; Niemegeers, I.G.M.M.; , "Cognitive Radio Emergency Networks - Requirements and Design," New Frontiers in Dynamic Spectrum Access Networks, 2005. DySPAN 2005. 2005 First IEEE International Symposium on , pp.601-606, Nov. 2005

[6] Kondareddy, Y.R.; Agrawal, P.; Sivalingam, K.; , "Cognitive Radio Network Setup Without a Common Control Channel," Military Communications Conference, 2008. MILCOM 2008. IEEE , pp.1-6, Nov. 2008

[7] Cordeiro, C.; Challapali, K.; , "C-MAC: A Cognitive MAC Protocol for Multi-Channel Wireless Networks," New Frontiers in Dynamic Spectrum Access Networks, 2007. DySPAN 2007. 2nd IEEE International Symposium on , vol., no., pp.147-157, Apr. 2007

[8] Jia, J.; Zhang, Q.; Shen, X.; , "HC-MAC: A Hardware-Constrained Cognitive MAC for Efficient Spectrum Management," Selected Areas in Communications, IEEE Journal on , vol.26, no.1, pp.106-117, Jan. 2008

[9] Zhao, Q.; Tong, L.; Swami, A.; Chen, Y.; , "Decentralized cognitive MAC for opportunistic spectrum access in ad hoc networks: A POMDP framework," Selected Areas in Communications, IEEE Journal on , vol.25, no.3, pp.589-600, April 2007

[10] Su, H.; Zhang, X.; , "Cross-Layer Based Opportunistic MAC Protocols for QoS Provisionings Over Cognitive Radio Wireless Networks," Selected Areas in Communications, IEEE Journal on , vol.26, no.1, pp.118-129, Jan. 2008

[11] M.Felegyhazi, M.Cagalj, P.Hubaux, "Efficient MAC in cognitive radio systems: A gametheoretic approach" in Wireless Communications IEEE Transactions, , vol. 8, pp. 1984 - 1995, Apr 2009

[12] Hamdaoui, B.; Shin, K.G.; , "OS-MAC: An Efficient MAC Protocol for Spectrum-Agile Wireless Networks," Mobile Computing, IEEE Transactions on , vol.7, no.8, pp.915-930, Aug. 2008

[13] IEEE Standard for Wireless LAN Medium Access Control (MAC) and Physical Layer (PHY) Specifications, Nov. 1997

[14] DaSilva, L.A.; Guerreiro, I.; , "Sequence-Based Rendezvous for Dynamic Spectrum Access," New Frontiers in Dynamic Spectrum Access Networks, 2008. DySPAN 2008. 3rd IEEE Symposium on , pp.1-7, 14-17 Oct. 2008

[15] Cormio, C; Chowdhury, K.R.; , "Common Control Channel Design for Cognitive Radio Wireless Ad-Hoc Networks Using Adaptive Frequency Hopping," Ad-Hoc Networks , pp. 430438, May. 2010

[16] Bianchi, G.; , "Performance Analysis of The IEEE 802.11 Distributed Coordination Function,", IEEE Journal on Selected Areas in Communications , vol.18, no.3, pp.535-547, Mar 2000

[17] Bianchi, G.; Tinnirello, I.; , "Remarks on IEEE 802.11 DCF performance analysis" IEEE Communications Letters, vol.9, no.8, pp. 765-767, Sep. 2005

[18] Akyildiz, I.F.; Lee, W.Y.; Chowdhury, K.R.; , "CRAHNs: Cognitive Radio Ad-Hoc Networks," Ad-Hoc Networks, vol.7, no.5, pp. 810-836, May. 2009 


\section{Authors}

Seyed Morteza Mirhoseini Nezhad received his B.Sc. degree in Computer Engineering (Hardware) from Shahid Bahonar University of Kerman, Iran, in 2008, and the M.Sc. degree in Computer Engineering (Computer Architecture) from the Iran University of Science and Technology (IUST), in 2011. He is currently continuing his researches in the Wireless Network Lab in Department of Computer Engineering at the IUST. The focus of his current research is on the various aspects of opportunistic spectrum access including spectrum sensing, management and cross-layer design of cognitive radio systems, and common control channel design of CRANs.

Reza Berangi received B.S. M.S. in Telecommunication Eng., From Iran University of Science and Technology, Tehran, Iran. The Ph.D. degree in Mobile Telecommunications from The University. He is currently an Associate Professor of Computer Engineering, Faculty of Computer Engineering, Iran University of Science and Technology. He is currently Assistant Professor of Computer Engineering faculty Iran University of Science and Technology, his research area includes Finding Mobility Management solutions for the next generation networks (NGN), wireless networks and communications systems, mobile computing, network protocol design and modeling.
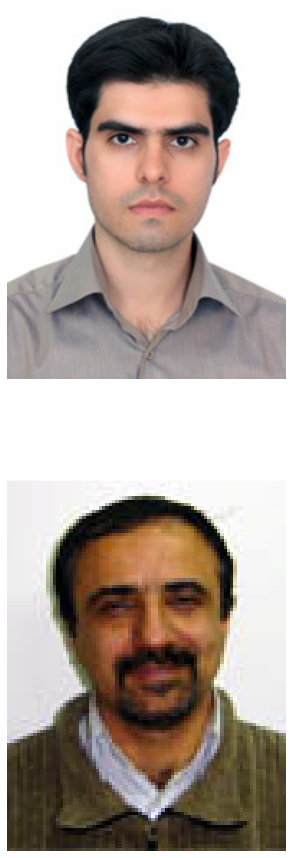

Mahmood Fathy received the B.S. degree in electronics from Iran University of Science and Technology, Tehran, Iran, in 1985, the M.S. degree in computer architecture from Bradford University, West Yorkshire, U.K., in 1987, and the Ph.D. degree in image processing computer architecture from the University of Manchester Institute of Science and Technology, Manchester, U.K., in 1991. Since 1991, he has been an Associate Professor with the Department of Computer Engineering, Iran University of Science and Technology. His research interests include the quality of service in computer networks, including video and image transmission over Internet, the applications of vehicular ad hoc networks in intelligent transportation systems, and real-time image processing, with particular interest in traffic engineering.

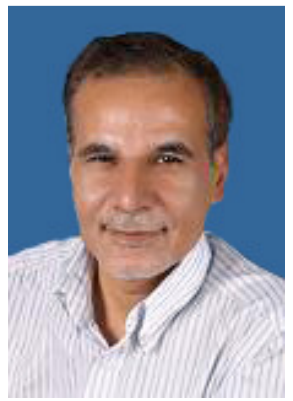

